\title{
Non-invasive markers of liver fibrosis in fatty liver disease are unreliable in people of South Asian descent
}

\author{
Sampath De Silva, ${ }^{1}$ Wenhao Li, ${ }^{1}$ Polychronis Kemos, ${ }^{1}$ James H Brindley, ${ }^{1}$ \\ Jibran Mecci, ${ }^{1}$ Salma Samsuddin, ${ }^{1}$ Joanne Chin-Aleong, ${ }^{2}$ Roger M \\ Feakins, ${ }^{2}$ Graham R Foster, ${ }^{1}$ Wing-Kin Syn, ${ }^{1,3,4}$ William Alazawi $^{1}$
}

\begin{abstract}
- Additional material is published online only. To view please visit the journal online (http://dx.doi.org/10.1136/ flgastro-2017-100865).

${ }^{1}$ Liver Unit, Blizard Institute, QueenMary University of London, London, UK

${ }^{2}$ Department of Histopathology, Bart's Health NHS Trust, London, UK

${ }^{3}$ Section of Gastroenterology, Ralph H Johnson Veterans Affairs Medical Center, Charleston, USA ${ }^{4}$ Division of Gastroenterology and Hepatology, Medical University of South Carolina, Charleston, USA
\end{abstract}

\section{Correspondence to} Dr William Alazawi, Liver Unit, Blizard Institute, QueenMary, University of London, E1 2AT, London; w.alazawi@qmul.ac.uk

Received 31 May 2017 Revised 1 August 2017 Accepted 19 August 2017 Published Online First 16 November 2017

\section{A) Check for updates}

To cite: De Silva S, Li W, Kemos P, et al. Frontline Gastroenterology

2018;9:115-121.

\section{ABSTRACT}

Objective Liver biopsy is the most accurate method for determining stage and grade of injury in non-alcoholic fatty liver disease (NAFLD). Given risks and limitations of biopsy, non-invasive tests such as NAFLD fibrosis score, aspartate transaminase (AST) to platelet ratio index, Fib-4, AST/alanine transaminase ratio and BARD are used. Prevalence and severity of NAFLD and metabolic syndrome vary by ethnicity, yet tests have been developed in largely white populations. We tested our hypothesis that noninvasive tests that include metabolic parameters are less accurate in South Asian compared with white patients.

Design Retrospective cross-sectional.

Setting Specialist liver centre.

Patients Patients with histologically confirmed NAFLD.

Interventions Scores calculated using clinical data taken within 1 week and compared with histology (Kleiner).

Main outcome measures Diagnostic test characteristics.

Results 175 patients were identified. South Asians ( $n=90)$ were younger, had lower body mass index and lower proportion of obesity compared with white patients $(n=79)$, with comparable rates of diabetes and liver injury. Tests are less sensitive at detecting advanced fibrosis in South Asian compared with white patients. Relative risk of correct diagnosis in white patients compared with South Asians is $1.86(95 \% \mathrm{Cl} 1.4$ to 2.6$)$. In binary logistic regression models, ethnicity and platelet count predicted accuracy. Transient elastography was equally and highly accurate in both ethnicities. Conclusions Blood test-based non-invasive scores are less accurate in South Asian patients, irrespective of metabolic parameters. Ethnicity should be considered when devising riskstratification algorithms for NAFLD.

\section{INTRODUCTION}

Non-alcoholic fatty liver disease (NAFLD) affects up to $30 \%$ of the general population $^{1}$ and is the hepatic manifestation of the metabolic syndrome. NAFLD is a spectrum of diseases that encompasses simple steatosis, non-alcoholic steatohepatitis (NASH) and fibrosis, which can lead to cirrhosis, liver failure and hepatocellular carcinoma. ${ }^{2}$ However, not all patients progress through the full hepatological spectrum of NAFLD. ${ }^{34}$ Determinants of progression include diabetes, diet and ethnicity but the most accurate predictor of liver-related mortality is presence of liver fibrosis on biopsy. ${ }^{5-7}$

It is neither feasible nor desirable to perform a liver biopsy in every patient with suspected NAFLD because the procedure is invasive, associated with potential complications, cost, sampling error and interobserver variability. Thus, non-invasive liver tests (NILTs) have been developed as an alternative to liver biopsy. These can be biomarker based ${ }^{8-10}$ or based on routinely collected clinical and laboratory data such as NAFLD fibrosis score (NFS), ${ }^{11}$ Fib-4, ${ }^{12}$ BARD, ${ }^{13}$ aspartate transaminase (AST) to platelet ratio index (APRI) and the AST/alanine transaminase (ALT) ratio. $^{14}$ Liver stiffness, measured by transient elastography (TE), ${ }^{15}$ acoustic radiation force impulse ${ }^{16}$ or MRI, ${ }^{17}$ can be a surrogate marker of fibrosis, but requires specialist equipment and/or skilled personnel to conduct the tests. 
Current guidance advocates use of these NILTs to stratify patients into specialist hepatology versus non-specialist or primary care. ${ }^{18}$ However, the applicability of blood test-based NILTs to different patient groups-including those of different ethnicitieshas yet to be determined. Most of the work done to develop and validate these scores has been in largely white Caucasian populations. ${ }^{11}$ Our group and others find that the prevalence of NAFLD varies by ethnic group with increased risk in patients of South Asian and Hispanic ethnicities. ${ }^{19-22}$ Diabetes is more common in South Asian patients and complications of obesity are evident at lower body mass indices. Diabetes and body mass index (BMI) contribute to the calculation of commonly used risk scores (NFS and BARD). Therefore, we hypothesised that these scores would be less accurate in patients of South Asian ethnicity compared with white patients, whereas there would be no difference in accuracy in tests that do not depend on these clinical factors: Fib-4, APRI, AST/ALT ratio and liver stiffness by TE. Surprisingly, we found reduced accuracy in all blood test-based scores but not TE in South Asian compared with white patients.

\section{PATIENTS AND METHODS}

We conducted a retrospective cross-sectional study of all adult patients with a histological diagnosis of any stage of NAFLD made in our centre between 2010 and 2016. The study was approved by the Barts Health National Health Service Trust Clinical Standards and Audit Department as a service evaluation of NILTs and therefore individual informed consent was not required or taken. Patients were excluded if they had any coexisting chronic liver disease, consumed more than 21 units $(168 \mathrm{~g})$ of alcohol per week for men and 14 units $(112 \mathrm{~g})$ of alcohol per week for women. Patients with inadequate biopsy specimens (as determined by the reporting pathologist), normal histology, alternative histological diagnoses or incomplete clinical data were excluded.

Liver biopsies reported by a single histopathologist (in routine clinical care) were summarised according to the National Institutes of Health NASH clinical research network (Kleiner) criteria. ${ }^{23}$ Clinical and laboratory data obtained within 1 week of the biopsy included sex, age, BMI (weight $(\mathrm{kg}) /$ height $\left(\mathrm{m}^{2}\right)$, alcohol consumption and diabetes status. Transient elastography was performed according to standard clinical practice. Data were obtained from clinical records and included if a valid result (successful reading rate $>60 \%$ and IQR of all readings $<30 \%$ of the median). Patients were classified as underweight, normal weight, overweight, obese or morbidly obese using BMI adjusted for ethnicity. ${ }^{24}$ We recorded self-reported ethnicity and collapsed results into four categories; South Asian (Indian, Pakistani, Bangladeshi, Sri Lankan and Nepalese), White, Black, East Asian and Other.
The NFS was calculated according to the formula: $-1.675+0.037 \times$ age $($ years $)+0.094 \times \mathrm{BMI}(\mathrm{kg} /$ $\left.\mathrm{m}^{2}\right)+1.13 \times$ impaired fasting glycaemia or diabetes $($ yes $=1$, no $=0)+0.99 \times$ AST $/$ ALT ratio $-0.013 \times$ platelet $\left(\times 10^{9} / \mathrm{L}\right)-0.66 \times$ albumin $(\mathrm{g} / \mathrm{dL})$. APRI was calculated as $\{($ AST (IU/l)/upper limit of normal)/platelet count $\left.\left(\times 10^{9}\right)\right\} \times 100$. The BARD score was calculated as the sum of three components (BMI $>28=1$ point, AST/ ALT ratio $>0.8=2$ points, diabetes $=1$ point). The BARD score was used as intended and so the BMI threshold was not adjusted for ethnicity. The Fib-4 score was calculated as: age $\times$ AST $(\mathrm{IU} / \mathrm{l}) /$ platelet count $\left(\times 10^{9} / \mathrm{L}\right)$.

The overall performance of NILTs was based on comparisons between the area under the receiveroperator curves (AUROC) for patients of South Asian and white ethnicities. Relative risk for sensitivity were calculated while controlling for choice of NILT. Cochran-Mantel-Haenszel statistics were used to calculate the common relative risk while the Breslow-Day statistic test was used to assess homogeneity. Analyses were performed in SAS (Cary, North Carolina, USA), Mintab (State College, Pennsylvania, USA) and SPSS (Armonk, New York, USA), and for all tests, the significance level was $\alpha=0.05$.

\section{RESULTS NASH is more aggressive in South Asian compared with white patients}

We identified 239 patients with NAFLD and no other liver diagnosis and, after excluding 64 because of inadequate specimens $(n=4)$ or incomplete data sets $(n=60)$, our cohort comprised 175 patients with biopsy-proven NAFLD and complete clinical data (table 1). The majority of patients were male $(\mathrm{n}=116,66 \%)$ and most were obese $(n=111,63 \%)$. Forty-six patients (26\%) had advanced fibrosis (stages 3 and 4), and 63 patients (36\%) had no fibrosis, of whom 26 (15\% of total) had steatosis alone.

The two largest ethnic groups in our cohort were South Asian $(n=90)$ and white $(n=79)$ patients (table 1). The remainder were of East Asian $(n=3)$, black $(n=2)$ or other $(n=1)$ ethnicities. Although there were no significant differences in the median grade or stage of liver injury, South Asians were almost a decade younger than white patients (43.6 vs 51.8 years, $\mathrm{p}=1.2 \times 10^{-5}$ ). The mean BMI of South Asian patients $\left(28.6 \mathrm{~kg} / \mathrm{m}^{2}\right)$ was lower than that of white patients $\left(32.8 \mathrm{~kg} / \mathrm{m}^{2}, \mathrm{p}=4.0 \times 10^{-8}\right)$. Using ethnicity-adjusted thresholds, ${ }^{24}$ proportionally fewer South Asian patients were obese $\left(60 \%\right.$ with BMI $\left.>27.5 \mathrm{~kg} / \mathrm{m}^{2}\right)$ compared with white patients $(75 \%$ with BMI $>30 \mathrm{~kg} /$ $\left.\mathrm{m}^{2}, \mathrm{p}<0.05\right)$.

Given this, it was not surprising that the mean NFS (which is calculated using both age and BMI) was significantly lower in South Asian patients (table 1, $\left.p=4.0 \times 10^{-4}\right)$. The same was true for Fib-4 $(p=0.01)$ but this did not reach statistical significance for AST/ 
Table 1 Demographic and clinicopathological characteristics of patients

\begin{tabular}{|c|c|c|c|c|c|}
\hline & \multicolumn{2}{|c|}{ South Asian $(n=90)$} & \multicolumn{2}{|l|}{ White $(n=79)$} & \multirow[b]{2}{*}{$p$ Value } \\
\hline & Mean/Median & Range & Mean/Median & Range & \\
\hline Age (years) & 44 & $24-78$ & 52 & $23-74$ & $1.2 \times 10^{-5}$ \\
\hline Sex (\%male) & $73 \%$ & & $61 \%$ & & 0.08 \\
\hline Diabetes (\%) & $42 \%$ & & $37 \%$ & & 0.52 \\
\hline $\mathrm{BMI}\left(\mathrm{kg} / \mathrm{m}^{2}\right)$ & 28.6 & $19-42$ & 32.8 & $23-52$ & $4.0 \times 10^{-8}$ \\
\hline \multicolumn{6}{|l|}{ Liver histology } \\
\hline Total NAS & 3 & $1-6$ & 3 & $1-6$ & 0.47 \\
\hline Steatosis & 1 & $1-3$ & 1 & $1-3$ & 0.89 \\
\hline Lobular inflammation & 1 & $0-2$ & 1 & $0-2$ & 0.01 \\
\hline Hepatocyte ballooning & 1 & $0-2$ & 1 & $0-2$ & 0.52 \\
\hline Fibrosis (Kleiner) & 1 & $0-4$ & 1 & $0-4$ & 0.60 \\
\hline $\begin{array}{l}\text { Fibrosis stage (n) } \\
\text { F0/F1/F2/F3/F4 }\end{array}$ & $34 / 23 / 11 / 15 / 17$ & & $28 / 23 / 5 / 12 / 11$ & & \\
\hline \multicolumn{6}{|l|}{ Blood results } \\
\hline AST (U/I) & 47 & $10-140$ & 48 & $16-133$ & 0.75 \\
\hline ALT (U/l) & 76 & $13-262$ & 69 & $12-288$ & 0.37 \\
\hline Platelets $\times 10^{9} / \mathrm{mL}$ & 237 & $62-449$ & 219 & $40-532$ & 0.11 \\
\hline Albumin (g/L) & 46 & $28-53$ & 45 & $33-52$ & 0.04 \\
\hline \multicolumn{6}{|l|}{ Non-invasive liver tests } \\
\hline NAFLD fibrosis score & -2.34 & $-5.8-3.4$ & -1.31 & $-6.1-3.0$ & $4.0 \times 10^{-4}$ \\
\hline APRI & 0.56 & $0.1-2.7$ & 0.65 & $0.2-3.3$ & 0.07 \\
\hline AST/ALT ratio & 0.70 & $0.3-2.0$ & 0.77 & $0.3-1.7$ & 0.13 \\
\hline BARD & 1 & $0-4$ & 1 & $0-4$ & 0.09 \\
\hline Fib-4 & 1.2 & $0.3-9.6$ & 1.81 & $0.5-10.5$ & 0.01 \\
\hline
\end{tabular}

Characteristics are summarised as mean for continuous data (age, BMI, blood results, NAFLD fibrosis score, APRI, AST/ALT and Fib-4) and as median for categorical data (histology and BARD score). Two-tailed p values compare South Asian and white patients using Student's t-test for continuous and the Mann-Whitney test for categorical data. Proportions of patients who are male and who have diabetes are compared using the $\chi^{2}$ test.

ALT, alanine aminotransferase; APRI, aspartate transaminase (AST) to platelet ratio index; AST, aspartate aminotransferase; BMI, body mass index;

NAFLD, non-alcoholic fatty liver disease; NAS, NAFLD activity score.

ALT, APRI or BARD. There was a positive association for each NILT with fibrosis score (see online supplementary figure 1) but the correlation was consistently higher in white patients than South Asians (see online supplementary table 1 ).
NILTs are less sensitive at detecting advanced fibrosis in South Asian patients

The AUROC was calculated for each test's ability to identify patients with advanced fibrosis (figure 1) using standard cut-off values $(\mathrm{NFS}>0.676$, APRI $>1.0$,

\section{A ROC of NILTs in South Asian Patients Advanced Fibrosis}

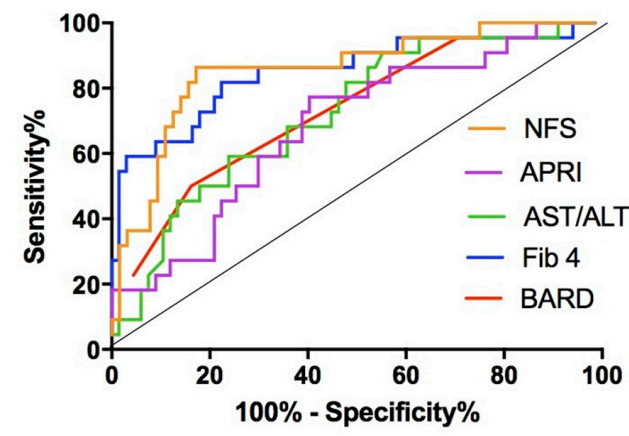

\section{B ROC of NILTs in White Patients Advanced Fibrosis}

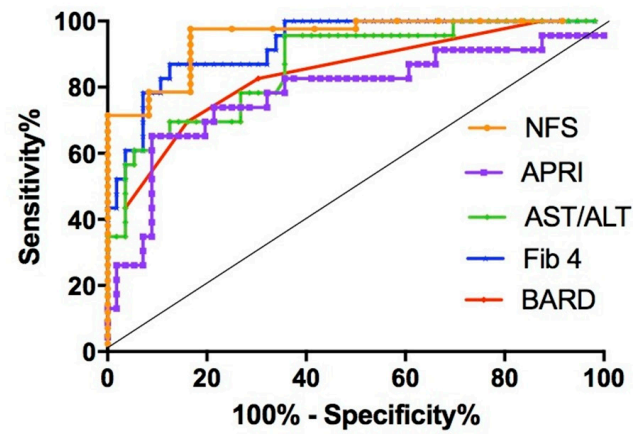

Figure 1 Receiver operating characteristic (ROC) curves for non-invasive liver tests. The diagnostic characteristics are shown for all five tests when used to assess advanced fibrosis (Kleiner stage 3 or 4) in patients of South Asian (A) or white (B) ethnicities. NFS, non-alcoholic fatty liver disease fibrosis score; APRI, aspartate aminotransferase to platelet ratio index, AST/ALT, aspartate aminotransferase to alanine aminotransferase ratio. 
AST/ALT ratio $>0.8$, BARD $>2$ and Fib-4 $>3.25$ ). In white patients, the NFS was most accurate at predicting advanced fibrosis (AUROC 0.95), followed by Fib-4, BARD, APRI and AST/ALT ratio (see online supplementary table 2). The AUROC for all tests was lower in South Asian compared with white patients (Friedman: $\mathrm{p}=0.025$ ) but the two most accurate scores in South Asians were still the NFS and Fib-4. Taken together, all five NILTs and not only those that contain components of the metabolic syndrome are less accurate in patients of South Asian compared with white ethnicity. NILTs were less sensitive in South Asian (range 0.09-0.52) compared with white patients (range $0.50-0.75$ ), but specificities were comparable.

Having confirmed homogeneity of relative risks, $(p=0.61$; Breslow-Day test), we found a strong association between ethnicity and NILT sensitivity $(p<0.0001$; Cochran-Mantel-Haenszel test). The relative risk of a true positive finding of advanced fibrosis is 1.85 (95\% CI 1.35 to 2.56) in white compared with South Asian patients (see online supplementary figure 2).

\section{Accuracy of NILTs is affected by ethnicity, platelet count and albumin}

To identify the factors that independently determine the accuracy of each NILT in our population, we performed analyses of association between a range of clinical and pathological parameters including ethnicity with correct diagnosis of fibrosis (see online supplementary table 3). Albumin and ALT or AST were significantly associated with correct diagnosis for all five NILTs. Other variables of note included platelet count, BMI and ethnicity. Ethnicity had a significant impact on accuracy of NFS and APRI, but the association with Fib-4 did not reach statistical significance $(p=0.065)$.

All variables that were significantly associated with NILT accuracy were considered for binary logistic regression models. The factors that predict the likelihood of the three most commonly used NILTs (NFS, APRI and Fib-4) in accurately detecting advanced fibrosis were platelet count, ethnicity and serum ALT (see online supplementary table 4). The impact of ethnicity was greatest on the NFS with borderline significance for an independent effect on APRI and Fib-4. There is a clear association of NILT accuracy with lower platelet counts and albumin levels (figure 2) although we did not include both in the regression models due to the strong correlation between both platelets and albumin levels with advanced fibrosis (and each other). NILTs (especially NFS, Fib-4 and APRI) are less likely to be accurate if the platelet count is over $150 \times 10^{9} / \mathrm{L}$ with $30 \%$ of tests correctly identifying advanced fibrosis compared with $69 \%$ in patients with platelet count below $150 \times 10^{9} / \mathrm{L}(\mathrm{p}<0.0001)$. Similarly, NILTs are less likely to be accurate if the albumin is $\geq 43 \mathrm{~g} / \mathrm{L}$ with $30 \%$ of tests correctly identifying advanced fibrosis compared with $68 \%$ in patients with albumin below $43 \mathrm{~g} / \mathrm{L}(\mathrm{p}<0.0001)$.
Transient elastography is more accurate than blood-based NILTs in South Asian patients

Data on TE (using Fibroscan) were available for a subset of our patients $(n=41)$. In South Asians, the AUROC for TE was much higher than other NILTs including NFS and Fib-4 $(\mathrm{p}=0.005)$. Using a liver stiffness of $7.9 \mathrm{kPa}$ to indicate indeterminate or high risk of advanced fibrosis, ${ }^{25} 26$ the sensitivity of TE for detecting advanced fibrosis was higher in both ethnic groups compared with blood test-based NILTs, while maintaining specificity above 90\% (figure 3 ).

\section{DISCUSSION}

The principal function of NILTs is to identify patients with high probability of having advanced fibrosis at the time of testing. Currently, decisions such as management in primary or secondary care or consideration for clinical trials are largely based on the results of these NILTs. As with any clinical test, there is a danger that if applied or interpreted inappropriately, wrong decisions can be made and, ultimately, harm may come to a patient. Despite the relatively small sample size, our data show that the sensitivity of commonly used NILTs is lower in patients of South Asian ethnicity suggesting that large numbers of South Asian patients may be inappropriately reassured that they do not have advanced disease. We recorded self-reported ethnicity and not first or subsequent generation immigration. Nevertheless, our data suggest that NASH may be more aggressive in South Asian patients who are almost a decade younger, have lower BMI and less severe BMI category than white patients with comparable disease stage. Although this has not been reported in South Asian patients before, our data are similar to those from Hispanic cohorts. ${ }^{21222728}$ Unfortunately, our patient cohort did not include sufficient numbers of patients of other Asian ethnic groups to comment on the differences between South Asian and, for example, East Asian ethnicities.

The factors that we identified on univariate analysis as being significantly associated with NILT accuracy (transaminases, BMI, platelet count, albumin and the presence of diabetes) are themselves components of NILT scores and are well known to be associated with an increased risk of advanced fibrosis. ${ }^{29}$ NILTs were designed and validated to identify patients with advanced fibrosis and it is therefore unsurprising that a NILT is more likely to be accurate if there is a priori evidence of advanced disease. For example, the heat maps in figure 2 show that NILTs are more likely to be accurate if the platelet count is below $150 \times 10^{9} / \mathrm{L}$ or albumin below $43 \mathrm{~g} / \mathrm{L}$. NILTs are able to identify patients who currently have cirrhosis or advanced fibrosis and who are at risk of developing complications such as hepatocellular carcinoma-essential tools for the primary care or general physician. However, there is no evidence to suggest that they can be used to identify patients with mild or moderate fibrosis in 


\begin{tabular}{|l|l|l|l|l|l|}
\hline Platelets & NFS & APRI & AST/ALT & BARD & Fib-4 \\
\hline 40 & & & & & \\
\hline 62 & & & & & \\
\hline 64 & & & & & \\
\hline 69 & & & & & \\
\hline 75 & & & & & \\
\hline 80 & & & & & \\
\hline 99 & & & & & \\
\hline 114 & & & & & \\
\hline 121 & & & & & \\
\hline 123 & & & & & \\
\hline 124 & & & & & \\
\hline 125 & & & & & \\
\hline 126 & & & & & \\
\hline 131 & & & & & \\
\hline 132 & & & & & \\
\hline 153 & & & & & \\
\hline 153 & & & & & \\
\hline 154 & & & & & \\
\hline 165 & & & & & \\
\hline 166 & & & & & \\
\hline 168 & & & & & \\
\hline 177 & & & & & \\
\hline 177 & & & & & \\
\hline 182 & & & & & \\
\hline 188 & & & & & \\
\hline 196 & & & & & \\
\hline 196 & & & & & \\
\hline 199 & & & & & \\
\hline 215 & & & & & \\
\hline 215 & & & & & \\
\hline 217 & & & & & \\
\hline 220 & & & & & \\
\hline 222 & & & & & \\
\hline 224 & & & & & \\
\hline 235 & & & & & \\
\hline 241 & & & & & \\
\hline 241 & & & & & \\
\hline 252 & & & & & \\
\hline 264 & & & & & \\
\hline 279 & & & & & \\
\hline 280 & & & & & \\
\hline 284 & & & & & \\
\hline 307 & & & & & \\
\hline 324 & & & & & \\
\hline 449 & & & & & \\
\hline
\end{tabular}

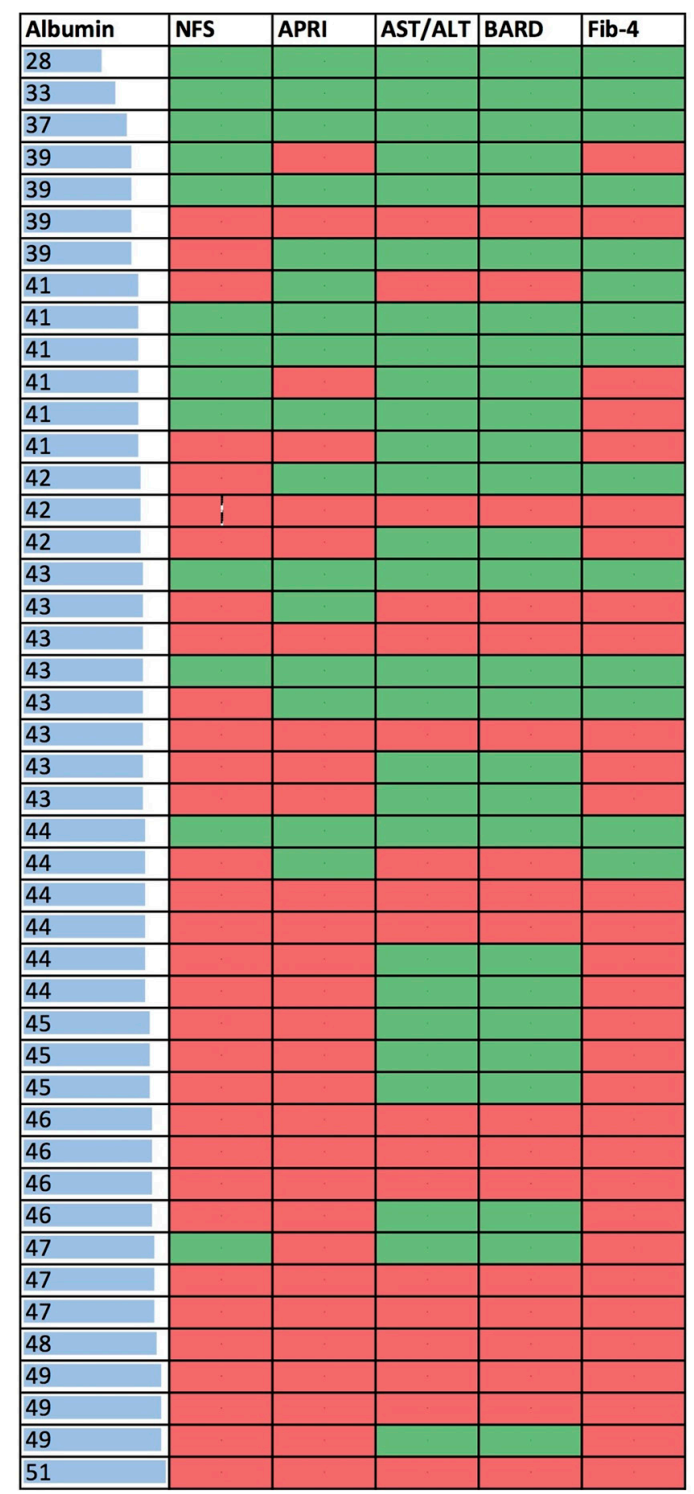

Figure 2 Heat maps showing the effect of platelets ( $\times 10^{9} / \mathrm{L}$; left) and albumin (g/L; right) on NILT accuracy in 46 patients of all ethnic groups with advanced fibrosis (Kleiner stage 3 or 4). Each row represents a single patient and shows the platelet count (left-hand heat map) or serum albumin concentration (right-hand heat map) is shown for that patient. Each column represents one of the five NILTs. A green cell in a column indicates that the test has correctly classified the patient as having advanced fibrosis and a red cell that the test has incorrectly classified the patient as not having advanced fibrosis. NFS, non-alcoholic fatty liver disease fibrosis score; APRI, aspartate aminotransferase to platelet ratio index, AST/ALT, aspartate aminotransferase to alanine aminotransferase ratio.

ROC: Transient elastography

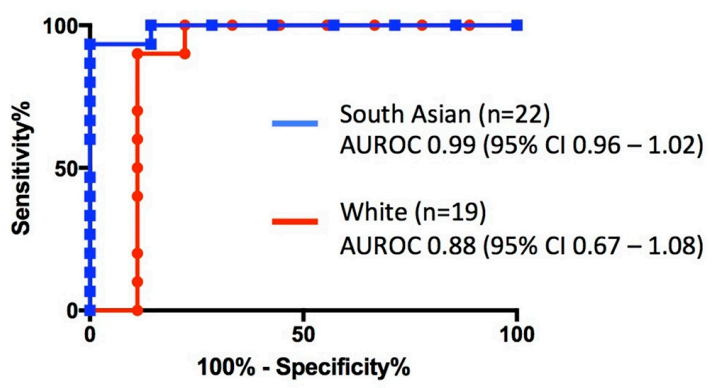

\begin{tabular}{|l|r|r|}
\hline & \multicolumn{2}{|c|}{$\begin{array}{c}\text { Transient } \\
\text { elastography }\end{array}$} \\
\hline & $\begin{array}{l}\text { South } \\
\text { Asian }\end{array}$ & White \\
\hline Sensitivity & 1.00 & 0.89 \\
\hline Specificity & 0.93 & 0.70 \\
\hline PPV & 0.88 & 0.73 \\
\hline NPV & 0.98 & 0.88 \\
\hline
\end{tabular}

0.88

Figure 3 Receiver operating characteristic (ROC) curves for transient elastography. Table shows test characteristics in white and South Asian patients. AUROC, area under the receiver-operator curves; NPV, negative predictive value; PPV, positive predictive value. 
whom current interventions (behaviour and lifestyle change) are most likely to be effective or who are the target population for the many clinical trials ongoing in NASH.

The overwhelming majority of patients in the original studies that derived NILTs were white (NFS 90\%, ${ }^{11}$ BARD $68 \%{ }^{13}$ and Fib-4 74\%). ${ }^{30}$ It is therefore unsurprising that we found comparable and high sensitivities and AUROCs in white patients with these NILTs. While some validation in populations of different ethnicities has been shown, ${ }^{31}$ little has been done in the South Asian population, who are at increased risk of metabolic syndrome.

In our analyses, we have used the accepted high cut-offs for NFS (>0.675) and Fib-4 (>3.25). Based on the AUROC curves in figure 1, it may be possible to improve the accuracy of these two NILTs (NFS and Fib-4) in South Asian patients. New thresholds can be determined that would maximise sensitivity while maintaining high specificity, even if not to the same degree as in white patients. These new cut-offs would need validation in an independent cohort as was done for patients aged over $65 .{ }^{32}$

We acknowledge that this is a single-centre study of retrospectively collected routine clinical data. As a result, not all patients had a fibroscan. The biopsies were performed and read by different operators with no review by a second pathologist. The histopathologists were not blinded to the clinical features or biochemical results when reporting these cases for routine clinical practice. We have not assessed the accuracy of biomarker-based tests such as $\operatorname{ELF}^{89}$ and Fibrotest ${ }^{10}$ that measure proteins involved in fibrosis and extracellular matrix turnover.

A variety of guidelines for the management of NAFLD have recently been published and the majority include the NILTs studied here. ${ }^{18} 33$ We have shown that NILTs may be inaccurate, for example, in patients with low pretest probability of significant disease or

\section{Key messages}

What is already known on this topic

- Many guidelines advocate the use of non-invasive tests to identify patients at high risk of advanced liver disease in non-alcoholic fatty liver disease.

\section{What this study adds}

- Blood test-based non-invasive liver tests, but not transient elastography, are less accurate in patients of South Asian compared with white ethnicity.

\section{How might it impact on clinical practice in the} foreseeable future

- Clinical guidance should take ethnicity into account when recommending assessment and treatment algorithms for non-alcoholic fatty liver disease. in patients of South Asian ethnicity; however, this is not the case for TE. We propose that ethnicity should be considered a factor in the clinical decision-making process. Further studies are required to validate and to assess the accuracy, utility and cost-effectiveness of new thresholds for NFS and Fib-4, and TE in patients with NAFLD.

Contributors SDS collected data and drafted the manuscript. WL, JHB, AJ and SS collected data. PK analysed results. JCA, RF, GRF and WA revised the manuscript. WA designed the study.

Funding Funded by the Diabetes Research and Wellness Foundation and supported by the North Thames Clinical Research Network. WA is in receipt of grants from the Medical Research Council and Liver and Pancreas Research UK.

Competing interests None declared.

Patient consent The study was approved by Barts Health NHS trust clinical standards and audit department as a service evaluation of NILTs and therefore specific informed consent was not required or taken.

Ethics approval Barts Health NHS Trust Clinical Standards and Audit Department.

Provenance and peer review Not commissioned; externally peer reviewed.

Open Access This is an Open Access article distributed in accordance with the terms of the Creative Commons Attribution (CC BY 4.0) license, which permits others to distribute, remix, adapt and build upon this work, for commercial use, provided the original work is properly cited. See: http://creativecommons.org/licenses/by/4.0/

(C) Article author(s) (or their employer(s) unless otherwise stated in the text of the article) 2018. All rights reserved. No commercial use is permitted unless otherwise expressly granted.

\section{REFERENCES}

1 Vernon G, Baranova A, Younossi ZM. Systematic review: the epidemiology and natural history of non-alcoholic fatty liver disease and non-alcoholic steatohepatitis in adults. Aliment Pharmacol Ther 2011;34:274-85.

2 Tai FW, Syn WK, Alazawi W. Practical approach to nonalcoholic fatty liver disease in patients with diabetes. Diabet Med 2015;32:1121-33.

3 Levene AP, Goldin RD. The epidemiology, pathogenesis and histopathology of fatty liver disease. Histopathology 2012;61:141-52.

4 McPherson S, Hardy T, Henderson E, et al. Evidence of NAFLD progression from steatosis to fibrosing-steatohepatitis using paired biopsies: implications for prognosis and clinical management. J Hepatol 2015;62:1148-55.

5 Angulo P. Long-term mortality in nonalcoholic fatty liver disease: is liver histology of any prognostic significance? Hepatology 2010;51:373-5.

6 Angulo P, Kleiner DE, Dam-Larsen S, et al. Liver fibrosis, but no other histologic features, is associated with long-term outcomes of patients with nonalcoholic fatty liver disease. Gastroenterology 2015;149:e10:389-397.

7 Chalasani N, Younossi Z, Lavine JE, et al. The diagnosis and management of non-alcoholic fatty liver disease: practice guideline by the american association for the study of liver diseases, american college of gastroenterology, and the american gastroenterological association. Hepatology 2012;55:2005-23.

8 Parkes J, Roderick P, Harris S, et al. Enhanced liver fibrosis test can predict clinical outcomes in patients with chronic liver disease. Gut 2010;59:1245-51. 
9 Rosenberg WM, Voelker M, Thiel R, et al. Serum markers detect the presence of liver fibrosis: a cohort study. Gastroenterology 2004;127:1704-13.

10 Poynard T, Imbert-Bismut F, Munteanu M, et al. Overview of the diagnostic value of biochemical markers of liver fibrosis (FibroTest, HCV FibroSure) and necrosis (ActiTest) in patients with chronic hepatitis C. Comp Hepatol 2004;3:8.

11 Angulo P, Hui JM, Marchesini G, et al. The NAFLD fibrosis score: a noninvasive system that identifies liver fibrosis in patients with NAFLD. Hepatology 2007;45:846-54.

12 Vallet-Pichard A, Mallet V, Nalpas B, et al. FIB-4: an inexpensive and accurate marker of fibrosis in HCV infection. comparison with liver biopsy and fibrotest. Hepatology 2007;46:32-6.

13 Harrison SA, Oliver D, Arnold HL, et al. Development and validation of a simple NAFLD clinical scoring system for identifying patients without advanced disease. Gut 2008;57:1441-7.

14 McPherson S, Stewart SF, Henderson E, et al. Simple noninvasive fibrosis scoring systems can reliably exclude advanced fibrosis in patients with non-alcoholic fatty liver disease. Gut 2010;59:1265-9.

15 Kwok R, Tse YK, Wong GL, et al. Systematic review with meta-analysis: non-invasive assessment of non-alcoholic fatty liver disease-the role of transient elastography and plasma cytokeratin-18 fragments. Aliment Pharmacol Ther 2014;39:254-69.

16 Liu H, Fu J, Hong R, et al. Acoustic radiation force impulse elastography for the non-invasive evaluation of hepatic fibrosis in non-alcoholic fatty liver disease patients: a systematic review \& meta-analysis. PLoS One 2015;10:e 0127782.

17 Loomba R, Wolfson T, Ang B, et al. Magnetic resonance elastography predicts advanced fibrosis in patients with nonalcoholic fatty liver disease: a prospective study. Hepatology 2014;60:1920-8.

18 European Association for the Study of the Liver (EASL)European Association for the Study of Diabetes (EASD)European Association for the Study of Obesity (EASO). EASL-EASD-EASO Clinical Practice Guidelines for the management of non-alcoholic fatty liver disease. J Hepatol 2016;64:1388-402.

19 Alazawi W, Mathur R, Abeysekera K, et al. Ethnicity and the diagnosis gap in liver disease: a population-based study. $\mathrm{Br} \mathrm{J}$ Gen Pract 2014;64:e694-e702.
20 Bambha K, Belt P, Abraham M, et al. Ethnicity and nonalcoholic fatty liver disease. Hepatology 2012;55:769-80.

21 Weston SR, Leyden W, Murphy R, et al. Racial and ethnic distribution of nonalcoholic fatty liver in persons with newly diagnosed chronic liver disease. Hepatology 2005;41:372-9.

22 Williams CD, Stengel J, Asike MI, et al. Prevalence of nonalcoholic fatty liver disease and nonalcoholic steatohepatitis among a largely middle-aged population utilizing ultrasound and liver biopsy: a prospective study. Gastroenterology 2011;140:124-31.

23 Kleiner DE, Brunt EM, Van Natta M, et al. Design and validation of a histological scoring system for non alcoholic fatty liver disease. Hepatology 2005;41:1313-21.

24 WHO Expert Consultation. Appropriate body-mass index for Asian populations and its implications for policy and intervention strategies. Lancet 2004;363:157-63.

25 Dyson JK, Anstee QM, McPherson S. Non-alcoholic fatty liver disease: a practical approach to diagnosis and staging. Frontline Gastroenterol 2014;5:211-8.

26 Wong VW, Vergniol J, Wong GL, et al. Diagnosis of fibrosis and cirrhosis using liver stiffness measurement in non alcoholic fatty liver disease. Hepatology 2010;51:454-62.

27 Browning JD, Szczepaniak LS, Dobbins R, et al. Prevalence of hepatic steatosis in an urban population in the United States: impact of ethnicity. Hepatology 2004;40:1387-95.

28 Schneider AL, Lazo M, Selvin E, et al. Racial differences in nonalcoholic fatty liver disease in the U.S. population. Obesity 2014;22:292-9.

29 Sharma S, Khalili K, Nguyen GC. Non-invasive diagnosis of advanced fibrosis and cirrhosis. World J Gastroenterol 2014;20:16820-30.

30 Sterling RK, Lissen E, Clumeck N, et al. Development simple noninvasive index to predict significant fibrosis in patients with HIV/HCV coinfection. Hepatology 2006;43:1317-25.

31 Sumida Y, Yoneda M, Hyogo H, et al. Validation of the FIB4 index in a Japanese nonalcoholic fatty liver disease population. BMC Gastroenterol 2012:2: 2.

32 McPherson S, Hardy T, Dufour JF, et al. Age as a confounding factor for the accurate Non-Invasive Diagnosis of Advanced NAFLD Fibrosis. Am J Gastroenterol 2017;112:74051.

33 Williams R, Aspinall R, Bellis M, et al. Addressing liver disease in the UK: a blueprint for attaining excellence in health care and reducing premature mortality from lifestyle issues of excess consumption of alcohol, obesity, and viral hepatitis. Lancet 2014;384:1953-97. 